\title{
BESCHÄFTIGUNGSQUOTEN NACH ALTERSGRUPPEN
}

Die einzelnen Arbeitsmärkte unterscheiden sich in Bezug darauf, wie die Beschäftigungsmöglichkeiten auf verschiedene Altersgruppen verteilt sind. Die Beschäftigungsquoten für Personen unterschiedlichen Alters werden in erheblichem Maße durch die staatliche Politik im Hinblick auf Tertiärbildung, Renten und Rentenalter beeinflusst.

\section{Definition}

Die Beschäftigungsquote einer bestimmten Altersgruppe wird als der Anteil der Erwerbstätigen dieses Alters an der Gesamtzahl der Personen in der jeweiligen Altersgruppe berechnet.

Die Erwerbstätigkeit wird in der Regel mittels nationaler Arbeitskräfteerhebungen gemessen. Nach dem ILO-Konzept gelten als Erwerbstätige Personen im Alter von 15 Jahren oder darüber, die angeben, dass sie in der Vorwoche mindestens eine Stunde erwerbstätig waren oder während der Referenz-

\section{Überblick}

Die Beschäftigungsquoten für Personen in der Altersgruppe 25-54 Jahre sind in den OECD-Ländern recht ähnlich: 2011 wurden in allen Ländern mit Ausnahme der Türkei Werte zwischen $68 \%$ und $87 \%$ verzeichnet. Größere Unterschiede im Ländervergleich sind bei Betrachtung der jüngsten Altersgruppe festzustellen, in der die Beschäftigungsquoten 2011 in neun Ländern - Griechenland, Ungarn, Italien, Slowakische Republik, Luxemburg, Korea, Spanien, Tschechische Republik und Polen - bei weniger als $26 \%$, aber in nur vier Ländern - Australien, Schweiz, Island und Niederlande - über $60 \%$ lagen. In der ältesten Altersgruppe variieren die Beschäftigungsquoten ebenfalls erheblich: zwischen $70 \%$ oder mehr in neun Ländern - Australien, Israel, Korea, Japan, Schweiz, Norwegen, Schweden, Neuseeland, Island - und weniger als $40 \%$ in acht Ländern - Slowenien, Türkei, Ungarn, Polen, Italien, Belgien, Luxemburg und Griechenland. In elf Ländern liegen die Beschäftigungsquoten der Personen im Haupterwerbsalter unter dem OECD-Durchschnitt - in Chile, Griechenland, Ungarn, Irland, Italien, Israel, Korea, Mexiko, Spanien, der Türkei und den Vereinigten Staaten -, wohingegen sie in Slowenien 8 Prozentpunkte über dem OECD-Durchschnitt liegen. In der Gruppe der aufstrebenden Volkswirtschaften liegen die Beschäftigungsquoten der jüngsten und der ältesten Altersgruppen nur in Brasilien über dem OECD-Durchschnitt, während die der Personen im Haupterwerbsalter in der Russischen Föderation um rd. 9 Prozentpunkte über dem OECD-Durchschnitt angesiedelt sind.

Infolge der andauernden Beschäftigungskrise sind die Beschäftigungsquoten der Personen im Haupterwerbsalter in einigen Ländern ganz erheblich gesunken; in Griechenland, Island, Irland, Spanien und den Vereinigten Staaten wurde ein Rückgang um 4 Prozentpunkte oder mehr verzeichnet. Die Beschäftigungsquoten älterer Arbeitskräfte sind im OECD-Durchschnitt um 6 Prozentpunkte gestiegen - selbst während der Beschäftigungskrise -, wobei die stärkste Zunahme in Neuseeland, den Niederlanden, Deutschland, Australien, Belgien und Finnland beobachtet wurde. woche in einem Beschäftigungsverhältnis standen, auch wenn dieses im fraglichen Zeitraum nicht ausgeübt wurde. Bei den Nichterwerbstätigen handelt es sich um Personen, die arbeitslos sind oder nicht am Erwerbsleben teilnehmen, wobei letzteres verschiedene Gründe haben kann: Studium, Schwierigkeiten bei der Arbeitssuche, Erwerbsunfähigkeit, Kindererziehung, Pflege älterer Familienangehöriger oder persönliche Präferenzen.

Die Beschäftigungsquoten werden für drei Altersgruppen dargestellt: für 15- bis 24-Jährige, d.h. Personen, die ihre Ausbildung gerade erst abgeschlossen haben und in den Arbeitsmarkt eintreten, für 25- bis 54-Jährige, d.h. Personen im Haupterwerbsalter, und für 55- bis 64-Jährige, d.h. Personen, die den Höhepunkt ihrer Karriere hinter sich haben und sich dem Rentenalter nähern.

\section{Vergleichbarkeit}

Die Beschäftigungsniveaus können durch Veränderungen in der Gestaltung und Durchführung der Erhebungen sowie Korrekturen der Kontrollvariablen auf der Basis von Zensusergebnissen und zwischen verschiedenen Erhebungswellen erstellten Zwischenschätzungen beeinflusst werden. Trotz dieser Abweichungen sind die hier dargestellten Beschäftigungsquoten im Zeitverlauf recht konsistent.

\section{Quelle}

- OECD (2012), OECD Employment Outlook, OECD Publishing.

- Für Nichtmitgliedsländer: Nationale Quellen.

Weitere Informationen

\section{Analysen}

- Burniaux, J.M., R. Duval und F. Jaumotte (2004), "Coping with Ageing", OECD Economics Department Working Papers, No. 371.

- OECD (2012), Bessere Kompetenzen, bessere Arbeitsplätze, ein besseres Leben: Ein strategischer Ansatz für die Kompetenzpolitik, OECD Publishing.

- OECD (2010), Off to a Good Start? Jobs for Youth, OECD Publishing.

- OECD (2006), Ageing and Employment Policies, OECD Publishing.

Statistiken

- OECD (2011), Labour Force Statistics, OECD Publishing.

Online-Datenbanken

- OECD Employment and Labour Market Statistics.

Websites

- OECD Ageing and Employment Policies (ergänzendes Material), www.oecd.org/els/employment/olderworkers.

- OECD Employment Policies, www.oecd.org/els/employment.

- OECD Jobs for Youth Project (ergänzendes Material), www.oecd.org/employment/youth.

- OECD Labour Statistics, www.oecd.org/std/labourstatistics. 


\section{ARBEITSMARKT • BESCHÄFTIGUNG UND GELEISTETE ARBEITSSTUNDEN}

BESCHÄFTIGUNGSQUOTEN NACH ALTERSGRUPPEN

Beschäftigungsquoten nach Altersgruppen

Erwerbstätige in Prozent der Bevölkerung der jeweiligen Altersgruppe

\begin{tabular}{|c|c|c|c|c|c|c|c|c|c|c|c|c|}
\hline & \multicolumn{4}{|c|}{ Erwerbstätige in der Altersgruppe 15-24 Jahre } & \multicolumn{4}{|c|}{ Erwerbstätige in der Altersgruppe 25-54 Jahre } & \multicolumn{4}{|c|}{ Erwerbstätige in der Altersgruppe 55-64 Jahre } \\
\hline & 1990 & 2000 & 2005 & 2011 & 1990 & 2000 & 2005 & 2011 & 1990 & 2000 & 2005 & 2011 \\
\hline Australien & 62.7 & 62.1 & 63.3 & 60.7 & 76.0 & 76.3 & 78.8 & 79.8 & 41.5 & 46.2 & 53.5 & 61.1 \\
\hline Belgien & 30.4 & 30.3 & 27.5 & 26.0 & 71.7 & 77.9 & 78.3 & 79.3 & 21.4 & 25.0 & 31.8 & 38.7 \\
\hline Chile & .. & 26.4 & 25.4 & 31.7 & .. & 65.0 & 67.5 & 74.2 & .. & 47.5 & 51.0 & 59.7 \\
\hline Dänemark & 65.0 & 67.1 & 62.3 & 57.5 & 84.0 & 84.3 & 84.5 & 82.3 & 53.6 & 54.6 & 59.5 & 59.5 \\
\hline Deutschland & 56.4 & 47.2 & 42.6 & 48.2 & 73.6 & 79.3 & 77.4 & 82.8 & 36.8 & 37.6 & 45.5 & 59.9 \\
\hline Estland & 51.7 & 32.9 & 29.8 & 32.3 & 91.8 & 75.7 & 79.3 & 78.1 & 60.4 & 44.0 & 55.7 & 57.1 \\
\hline Finnland & 55.2 & 42.9 & 42.1 & 42.3 & 87.9 & 80.9 & 81.7 & 82.3 & 42.8 & 42.3 & 52.6 & 57.0 \\
\hline Frankreich & 35.7 & 28.3 & 30.2 & 29.9 & 77.3 & 78.4 & 80.7 & 81.3 & 30.7 & 29.3 & 38.5 & 41.4 \\
\hline Griechenland & 30.3 & 26.9 & 25.0 & 16.3 & 68.5 & 70.2 & 74.0 & 69.0 & 40.8 & 39.0 & 41.6 & 39.4 \\
\hline Irland & 41.4 & 49.4 & 47.9 & 28.1 & 60.0 & 75.5 & 77.8 & 69.8 & 38.6 & 45.2 & 51.6 & 50.8 \\
\hline Island & .. & 68.2 & 71.6 & 63.3 & .. & 90.6 & 88.2 & 84.0 & .. & 84.2 & 84.8 & 79.5 \\
\hline Israel & 23.6 & 28.2 & 26.6 & 26.6 & 66.5 & 70.4 & 70.6 & 74.8 & 48.5 & 46.6 & 52.4 & 61.2 \\
\hline Italien & 29.8 & 27.8 & 25.5 & 19.4 & 68.2 & 68.0 & 72.2 & 71.1 & 32.6 & 27.7 & 31.4 & 37.9 \\
\hline Japan & 42.2 & 42.7 & 40.9 & 39.1 & 79.6 & 78.6 & 79.0 & 80.2 & 62.9 & 62.8 & 63.9 & 65.1 \\
\hline Kanada & 61.3 & 56.2 & 57.7 & 55.4 & 78.1 & 79.9 & 81.3 & 81.0 & 46.2 & 48.1 & 54.7 & 58.7 \\
\hline Korea & 32.5 & 29.4 & 29.9 & 23.1 & 73.2 & 72.2 & 73.4 & 74.4 & 61.9 & 57.8 & 58.7 & 62.1 \\
\hline Luxemburg & 43.3 & 31.8 & 24.9 & 20.7 & 71.8 & 78.2 & 80.7 & 82.0 & 28.2 & 27.2 & 31.7 & 39.3 \\
\hline Mexiko & .. & 48.9 & 43.7 & 42.0 & .. & 67.4 & 68.8 & 69.5 & .. & 51.7 & 52.6 & 53.4 \\
\hline Neuseeland & 59.1 & 54.2 & 56.4 & 49.9 & 76.3 & 78.3 & 81.6 & 80.4 & 41.8 & 56.9 & 69.5 & 73.7 \\
\hline Niederlande & 54.5 & 66.5 & 61.7 & 63.6 & 71.2 & 81.0 & 81.5 & 84.2 & 29.7 & 37.6 & 44.8 & 56.1 \\
\hline Norwegen & 53.4 & 58.1 & 52.9 & 51.4 & 82.2 & 85.3 & 83.2 & 84.7 & 61.5 & 67.1 & 67.6 & 69.6 \\
\hline Österreich & .. & 52.8 & 53.1 & 54.9 & .. & 82.5 & 82.6 & 84.9 & .. & 28.3 & 31.8 & 41.5 \\
\hline Polen & .. & 24.5 & 20.9 & 24.9 & .. & 70.9 & 69.5 & 77.2 & .. & 28.4 & 29.1 & 36.9 \\
\hline Portugal & 54.8 & 41.8 & 36.1 & 27.1 & 78.4 & 81.8 & 80.8 & 77.8 & 47.0 & 50.7 & 50.5 & 47.9 \\
\hline Schweden & 66.1 & 46.7 & 43.3 & 40.4 & 91.6 & 83.8 & 83.9 & 86.0 & 69.5 & 65.1 & 69.6 & 72.5 \\
\hline Schweiz & .. & 65.1 & 59.9 & 62.9 & .. & 85.4 & 85.1 & 86.4 & .. & 63.3 & 65.1 & 69.5 \\
\hline Slowak. Rep. & .. & 29.0 & 25.6 & 20.2 & .. & 74.7 & 75.3 & 76.5 & .. & 21.3 & 30.4 & 41.4 \\
\hline Slowenien & .. & .. & 34.1 & 31.5 & .. & .. & 83.8 & 83.1 & .. & .. & 30.7 & 31.2 \\
\hline Spanien & 38.3 & 36.3 & 41.9 & 24.1 & 61.4 & 68.4 & 74.4 & 68.7 & 36.9 & 37.0 & 43.1 & 44.5 \\
\hline Tschech. Rep. & .. & 38.3 & 27.3 & 24.7 & .. & 81.6 & 82.0 & 82.8 & .. & 36.3 & 44.6 & 47.6 \\
\hline Türkei & 45.9 & 37.0 & 30.2 & 32.1 & 61.6 & 56.7 & 53.0 & 57.5 & 42.7 & 36.4 & 28.0 & 31.4 \\
\hline Ungarn & .. & 32.5 & 21.8 & 18.3 & .. & 73.0 & 73.7 & 73.1 & .. & 21.9 & 33.0 & 35.8 \\
\hline Ver. Königreich & 70.1 & 61.5 & 58.7 & 50.1 & 79.1 & 80.2 & 81.1 & 80.1 & 49.2 & 50.4 & 56.7 & 56.8 \\
\hline Ver. Staaten & 59.8 & 59.7 & 53.9 & 45.5 & 79.7 & 81.5 & 79.3 & 75.1 & 54.0 & 57.8 & 60.8 & 60.0 \\
\hline EU27 & .. & .. & .. &.. & .. & .. & .. &.. & .. & .. & .. & .. \\
\hline OECD & 49.1 & 45.5 & 42.7 & 39.5 & 75.8 & 75.9 & 75.8 & 75.4 & 47.7 & 47.6 & 51.7 & 54.4 \\
\hline Brasilien & .. & .. & 52.7 &.. & .. & .. & 75.9 &.. & .. & .. & 54.1 & .. \\
\hline China & .. & .. & .. &.. & .. & .. & .. &.. & .. & .. & .. & .. \\
\hline Indien & .. & .. & .. &.. & .. & .. & .. &.. & .. & .. & .. & .. \\
\hline Indonesien & .. &.. & .. &.. & .. & .. & .. & .. & .. & .. & .. & .. \\
\hline Russ. Föderation & .. & 34.3 & 32.5 & 35.8 & .. & 79.6 & 82.6 & 84.6 & .. & 34.6 & 44.3 & 43.8 \\
\hline Südafrika & .. & .. & 15.0 & 12.7 & .. & .. & 59.3 & 56.5 & .. & .. & 42.2 & 38.0 \\
\hline
\end{tabular}

Beschäftigungsquoten in der Altersgruppe 15-24 Jahre

Erwerbstätige in Prozent der Bevölkerung dieser Altersgruppe

$$
2011 \quad \diamond 2000
$$

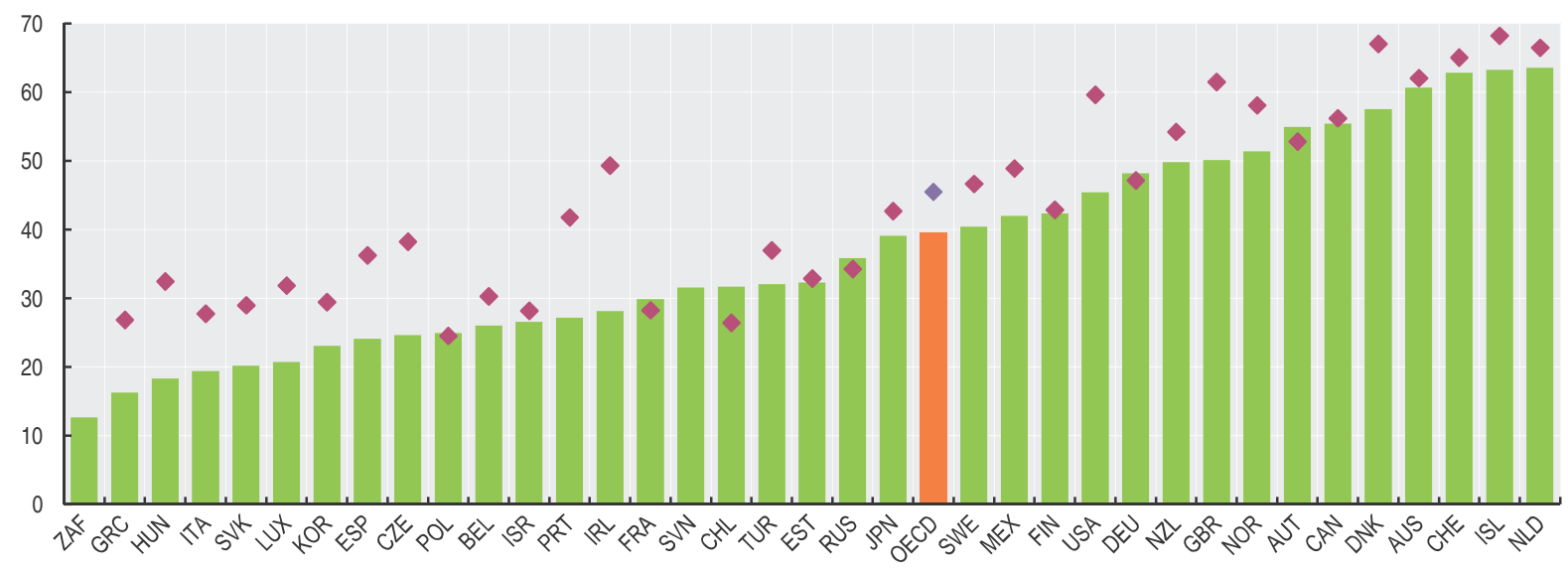




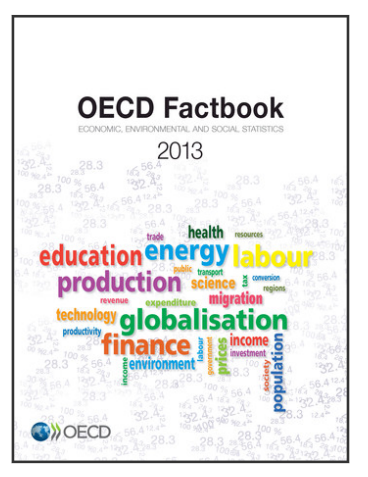

\section{From: \\ OECD Factbook 2013 \\ Economic, Environmental and Social Statistics}

Access the complete publication at:

https://doi.org/10.1787/factbook-2013-en

Please cite this chapter as:

OECD (2013), "Beschäftigungsquoten nach Altersgruppen", in OECD Factbook 2013: Economic, Environmental and Social Statistics, OECD Publishing, Paris.

DOI: https://doi.org/10.1787/factbook-2013-52-de

Das vorliegende Dokument wird unter der Verantwortung des Generalsekretärs der OECD veröffentlicht. Die darin zum Ausdruck gebrachten Meinungen und Argumente spiegeln nicht zwangsläufig die offizielle Einstellung der OECDMitgliedstaaten wider.

This document and any map included herein are without prejudice to the status of or sovereignty over any territory, to the delimitation of international frontiers and boundaries and to the name of any territory, city or area.

You can copy, download or print OECD content for your own use, and you can include excerpts from OECD publications, databases and multimedia products in your own documents, presentations, blogs, websites and teaching materials, provided that suitable acknowledgment of OECD as source and copyright owner is given. All requests for public or commercial use and translation rights should be submitted to rights@oecd.org. Requests for permission to photocopy portions of this material for public or commercial use shall be addressed directly to the Copyright Clearance Center (CCC) at info@copyright.com or the Centre français d'exploitation du droit de copie (CFC) at contact@cfcopies.com. 